\title{
Brain-Derived Neurotrophic Factor Modulates Auditory Function in the Hearing Cochlea
}

\author{
David J. Sly, ${ }^{1}$ Amy J. Hampson, ${ }_{1}^{1}$ Ricki L. Minter, ${ }^{1}$ LeOn F. Heffer,${ }^{1}$ Jack Li, ${ }^{1}$ Rodney E. Millard, ${ }^{1}$ \\ Leon Winata, ${ }^{1}$ Allen Niasari, ${ }^{1}$ and Stephen J. O’Leary ${ }^{1}$ \\ ${ }^{1}$ Department of Otolaryngology, University of Melbourne, 32 Gisborne Street, Melbourne, 3002, Victoria, Australia
}

Received: 3 February 2011; Accepted: 13 October 2011; Online publication: 16 November 2011

\begin{abstract}
Neurotrophins prevent spiral ganglion neuron (SGN) degeneration in animal models of ototoxin-induced deafness and may be used in the future to improve the hearing of cochlear implant patients. It is increasingly common for patients with residual hearing to undergo cochlear implantation. However, the effect of neurotrophin treatment on acoustic hearing is not known. In this study, brain-derived neurotrophic factor (BDNF) was applied to the round window membrane of adult guinea pigs for 4 weeks using a cannula attached to a mini-osmotic pump. SGN survival was first assessed in ototoxically deafened guinea pigs to establish that the delivery method was effective. Increased survival of SGNs was observed in the basal and middle cochlear turns of deafened guinea pigs treated with BDNF, confirming that delivery to the cochlea was successful. The effects of BDNF treatment in animals with normal hearing were then assessed using distortion product otoacoustic emissions (DPOAEs), pure tone, and clickevoked auditory brainstem responses (ABRs). DPOAE assessment indicated a mild deficit of $5 \mathrm{~dB}$ SPL in treated and control groups at 1 and 4 weeks after cannula placement. In contrast, ABR evaluation showed that BDNF lowered thresholds at specific frequencies $(8$ and $16 \mathrm{kHz}$ ) after 1 and 4 weeks posttreatment when compared to the control cohort receiving Ringer's solution. Longer treatment for 4 weeks not only widened the range of frequencies ameliorated from 2
\end{abstract}

David Sly and Amy Hampson contributed equally to this work (co-first authors).

Correspondence to: David J. Sly · Department of Otolaryngology · University of Melbourne · 32 Gisborne Street, Melbourne, 3002, Victoria, Australia. Telephone: +1-613-99298385; fax: +1-61396631958dsly@unimelb.edu.au to $32 \mathrm{kHz}$ but also lowered the threshold by at least $28 \mathrm{~dB}$ SPL at frequencies $\geq 16 \mathrm{kHz}$. BDNF treatment for 4 weeks also increased the amplitude of the ABR response when compared to either the control cohort or prior to treatment. We show that BDNF applied to the round window reduces auditory thresholds and could potentially be used clinically to protect residual hearing following cochlear implantation.

Keywords: neurotrophins, residual hearing, cochlear implant, guinea pig, auditory brainstem response, otoacoustic emissions

\section{INTRODUCTION}

Cochlear implant function may be improved by achieving greater electrode selectivity, bringing electrodes and neurons into closer proximity so that a greater number of electrodes could be used, with each stimulating a smaller number of auditory neurons (O'Leary et al. 2009). Neurotrophins have been pivotal in research directed towards achieving this aim. These proteins have been shown to rescue auditory neurons from cell death after sensorineural hearing loss (Staecker et al. 1996, 1998; Miller et al. 1997; Gillespie et al. 2004) and to promote the regrowth of the peripheral dendrites of surviving neurons (Wise et al. 2005; Miller et al. 2007; Evans et al. 2009) with both effects being desirable to increase electrode specificity (Miller et al. 2007; O'Leary et al. 2009).

The broadening of cochlear implant candidacy to include patients with residual hearing (Gantz et al. 2005) means that the possible effects of neurotrophins upon acoustically driven cochlear function must be considered. Such investigations will extend the current literature, which suggests that neurotrophins 
are likely to be safe in a deafened cochlea when delivered exogenously with polymeric gels (Noushi et al. 2005; Havenith et al. 2011) or miniature osmotic pumps (Miller et al. 1997; Shepherd et al. 2005). Functional studies in deafened animals treated with neurotrophins reveal that electrically evoked auditory brainstem response (ABR) thresholds are lowered (Shinohara et al. 2002; Shepherd et al. 2005; Richardson et al. 2009) and may be reduced below pretreatment levels. However, because the ABR is a population response, it is not clear whether this effect is due to a change in threshold of individual auditory neurons, the greater neuronal survival that accompanies neurotrophin application to the deafened ear, or possibly tighter neural synchrony. The present study may provide some further insights into these questions as spiral ganglion neurons (SGNs) are not expected to degenerate in hearing ears treated with neurotrophins.

Here we explore the effects of brain-derived neurotrophic factor (BDNF) upon acoustically driven hearing. Achieving this aim requires the development of a method for delivering BDNF to the inner ear without causing substantial hearing loss. This presented a challenge given that most available delivery systems, such as intrascalar infusion or viral transfection, involve cochlear surgery that carries a significant risk to hearing. For example, Agterberg et al. (2008) reported substantial damage to both inner hair cells (25\% remaining) and outer hair cells (42\% remaining) at the lower basal turn of the cochlea after intrascalar infusion of BDNF in normal hearing guinea pigs. Polymers, particularly hydrogels that may be applied directly to the round window, were considered (Noushi et al. 2005). However, most have a release profile that varies over time, so instead we chose to place a cannula attached to a mini-osmotic pump directly onto the round window. It was first necessary to estimate to what extent BDNF was distributed in the cochlea using a cannula-based delivery system. This was achieved by determining the extent to which BDNF, when delivered to the round window of deafened guinea pigs, enhanced SGN survival. In subsequent experiments, BDNF was delivered to guinea pigs with normal hearing and the effects upon auditory function were evaluated.

\section{METHODS}

All procedures reported in this study were approved by the Animal Research and Ethics Committee of the Royal Victorian Eye and Ear Hospital (Project \# 08/ 159) and performed in accordance with the National Health and Medical Research Council's 'Australian Code of Practice for the Care and Use of Animals for Scientific Purposes'.

\section{Experimental subjects and design}

Twenty-four adult Dunkin-Hartley pigmented guinea pigs (15 males, 9 females; body weight 233-815 g) were obtained from our in-house breeding centre and randomly allocated into one of four experimental groups, all of which received treatments for 4 weeks to the left cochlea. To ensure that the round window delivery method was effective, 12 animals were bilaterally deafened 1 week prior to cannula placement. Six of these animals then received BDNF treatment and six received Ringer's solution to serve as deafened controls. BDNF was chosen because it has been shown to improve SGN survival after deafness in several studies in the guinea pig (Staecker et al. 1996; Miller et al. 1997; Gillespie et al. 2004; Shepherd et al. 2005; Agterberg et al. 2008) and this was a requirement of the validation of the round window delivery method developed here. Furthermore, BDNF has been the most extensively examined neurotrophin for potential use with cochlear implants (Shepherd et al. 2005, 2008; Song et al. 2009); thus, it seemed appropriate to begin our investigations in hearing animals with this protein.

Once treatment had concluded, the animals were euthanized and the round window membrane was exposed. Fluid was then manually pushed through the delivery system with a syringe to test its patency in situ. Cochleae were then removed and SGN density was assessed to establish whether our delivery method resulted in the usual protection of SGNs observed with BDNF treatment (Staecker et al. 1996; Miller et al. 1997; Gillespie et al. 2004; Shepherd et al. 2005; Wise et al. 2005; Agterberg et al. 2008; Havenith et al. 2011).

The remaining 12 guinea pigs served as a normal hearing cohort. Pure tone and click-evoked ABRs were conducted on this group to check hearing levels across a range of frequencies and distortion product otoacoustic emissions (DPOAEs) were used to assess the function of outer hair cells. Five of these animals received BDNF treatment and seven received Ringer's solution to act as normal hearing controls. ABRs and DPOAEs were recorded immediately prior to cannula implantation and repeated at 1 and 4 weeks after surgery to assess the effect of BDNF treatment on normal hearing. Following the final ABR recording, the patency of the delivery system was tested as described below and the cochleae were removed for histological analysis.

\section{Animal care}

All experimental procedures were conducted under general anaesthesia using an intramuscular injection of ketamine $(60 \mathrm{mg} / \mathrm{kg}$ for induction, $40 \mathrm{mg} / \mathrm{kg}$ for 
maintenance; Ketamil, Troy Laboratories Pty. Ltd., Smithfield, NSW, Australia) and xylazine (4 mg/kg; Xylazil-20, Troy Laboratories), and the animals were maintained in an areflexic state. Body temperature was maintained at $37-39^{\circ} \mathrm{C}$ using a custom-made direct-current heating pad; ocular ointment (Novartis Pharmaceuticals Australia Pty. Ltd., North Ryde, NSW, Australia) was applied generously to the eyes and fluid replacement was given via subcutaneous injections of Hartmann's solution $(10 \mathrm{~mL} / \mathrm{kg})$. The local anaesthetic lignocaine $(20 \mathrm{mg} / \mathrm{kg}$; Lignocaine-20, Troy Laboratories), delivered subcutaneously, was used for skin incisions.

\section{Auditory brainstem response recordings}

Prior to experimentation (pretreatment), the hearing status of each animal was assessed to ensure that they all had normal hearing using a click-evoked ABR, using criteria that are well established in our laboratories (Shepherd and Clark 1985; Hellier et al. 2002; Wise et al. 2005; Sly et al. 2007; Heffer et al. 2010). Animals were considered to have normal hearing if a stimulus intensity of $\leq 48 \mathrm{~dB}$ SPL peak equivalent to $100 \mu$ s negative rarefaction click, delivered free field, evoked wave III of the ABR greater than $0.4 \mu \mathrm{V}$. The wave amplitude was chosen as it exceeds the noise floor. Wave III was analysed as this is the basis for accepting normalcy of hearing in our laboratories. Furthermore, wave III can be detected at lower stimulation levels than earlier waves, especially when evaluating the response to tone pips.

In normal hearing animals, ABR recordings were made pretreatment, immediately postsurgery, and at 1 and 4 weeks posttreatment. Recordings were made from subcutaneous needle electrodes placed at the vertex and the nape of the neck, with a ground electrode placed more caudally. The contralateral ear was occluded using an ear mould compound (Otoform; Dreve, Unna, Germany). Pure tone pips lasting $5 \mathrm{~ms}$ with $1 \mathrm{~ms}$ linear rise/fall time were presented over 250 repetitions at a rate of 33 repetitions per second at frequencies of $0.25,0.5,1,2,4,8,16$ and $32 \mathrm{kHz}$, delivered through a loudspeaker (Peerless, Mumbai, India) placed $10 \mathrm{~cm}$ away from the ear in line with the external auditory meatus. For each frequency, stimulus intensity was decreased in $5 \mathrm{~dB}$ steps from $100 \mathrm{~dB}$ SPL to subthreshold levels.

Responses were differentially amplified with a gain of 1,000 (DAM-5A; World Precision Instruments, Sarasota, FL, USA) and band-pass filtered between $150 \mathrm{~Hz}$ (24 dB/octave) and $3 \mathrm{kHz}$ (6 dB/octave) using a 375OR filter (Krohn-Hite Corporation, Brockton, MA, USA). Responses were further amplified with a gain of 100 with a custom-made amplifier. This output was digitized with a 10-bit analogue to digital convert- er (Tucker Davis Technologies, Alachua, FL, USA) sampling at $20 \mathrm{kHz}$ for $12.5 \mathrm{~ms}$ following the stimulus onset. Responses were averaged over 250 stimulus repetitions. The waveforms were exported to a custom software routine in Igor Pro 6.0 (Wavemetrics, Inc., Portland, OR, USA) and analysed to isolate wave III of the response. The threshold, amplitude, and peak latency of wave III were determined and compared for the effect of treatment across duration, at pretreatment, at 1 week and 4 weeks after treatment and across stimulus frequencies. Threshold was defined as the lowest-intensity stimulus which evoked a wave III response greater than $0.4 \mu \mathrm{V}$ in amplitude.

\section{Otoacoustic emission recordings}

DPOAEs were recorded in all normal hearing guinea pigs. Stimuli were delivered using a Smart DPOAE system (Intelligent Hearing Systems, Miami, FL, USA) coupled to high-frequency transducers (Intelligent Hearing Systems) and a combination sound delivery/microphone probe (10B+, Etymotic Research, Elk Grove Village, IL, USA). The probe was fitted into the external auditory meatus of the guinea pig with a silicone probe tip to form a closed-field delivery and recording system.

According to typical DPOAE protocols, two puretone stimuli, at frequencies F1 and F2, were delivered through the probe. In these studies, F1 stimuli were delivered between frequencies of 0.25 and $32 \mathrm{kHz}$ in three steps per octave, where $\mathrm{F} 1 / \mathrm{F} 2=1.3$ and where the stimulus intensity of $\mathrm{F} 1=\mathrm{F} 2$. To reduce noise floor, recordings were made from four blocks with 32 sweeps in each block. Recordings were made at intensity levels of 45, 55, 65 and $75 \mathrm{~dB}$ SPL. These settings were based both on our own trials and on previous studies (Polak 2004) to obtain the largest DPOAE responses. The DPOAE response generated at $2 \mathrm{~F} 1-\mathrm{F} 2$ was used in analyses by plotting the amplitude of the response against the F1/F2 stimulus frequency. These responses were compared for the effects of treatment across time and frequency.

\section{Deafening and round window drug administration}

Guinea pigs were bilaterally deafened with a single dose of kanamycin sulphate $(400 \mathrm{mg} / \mathrm{kg}$; SigmaAldrich, St Louis, MO, USA) administered subcutaneously and frusemide (100 mg/kg; Troy Laboratories) administered intravenously. One week later, all guinea pigs were confirmed deaf by testing ABRs. Deafness was defined as a zero amplitude wave III response to a $100-\mu$ s rarefaction click presented at $100 \mathrm{~dB}$ SPL.

All treatments involved chronic delivery of either BDNF or Ringer's solution to the left cochlea through an implanted delivery system (Fig. 1). Solutions were 


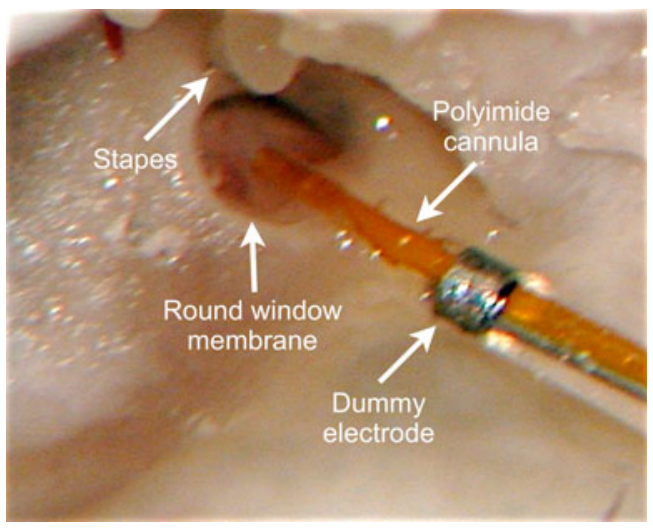

FIG. 1. Drug delivery to the round window membrane. A polyimide cannula connected to PVC tubing was placed near the intact round window membrane to deliver chronic drug treatments to the cochlea. A dummy electrode was used to guide the depth of insertion of the cannula into the bulla. The cannula was connected to a mini-osmotic pump (not shown) located under the skin at the scapulae and the pump delivered treatments over 4 weeks.

administered using a custom drug delivery cannula, the tip of which was placed carefully on the lip of the round window niche. The cannula was made from PVC tubing adapted to a narrow polyimide cannula (Miller et al. 1997; Gillespie et al. 2004). The cannula was attached to a mini-osmotic pump (Alzet model \#2004; Durect Corporation, Cupertino, CA, USA) with a reservoir capacity of $200 \mu \mathrm{L}$ and a flow rate of $0.25 \mu \mathrm{L} / \mathrm{h}$ over 28 days. BDNF treatment was delivered at a concentration of $62.5 \mu \mathrm{g} / \mathrm{mL}$ (Recombinant Human BDNF, Peprotech, Rocky Hill, NJ, USA) in Ringer's solution. All mini-osmotic pumps were prepared under sterile conditions in a clean laminar flow hood 1 day prior to surgery and incubated at $37^{\circ} \mathrm{C}$ overnight. The BDNF concentration chosen has been shown to promote SGN survival in deafened guinea pigs in previous studies (Gillespie et al. 2003; Shepherd et al. 2005; Wise et al. 2005), which meant that in the present study SGN survival in the deafened animals could be used to assess the success of the round window delivery method. When radiolabelled neurotrophin-3 is applied at similar concentrations directly into the basal cochlear turn, it is detected over the lower two cochlear turns (Richardson et al. 2004).

Prior to surgery for implantation of the cannula, guinea pigs received the antibiotic enrofloxacin (20 mg/kg; Baytril, Bayer Healthcare, Leverkusen, Germany), administered subcutaneously. All implant surgeries were performed under general anaesthesia and strict aseptic conditions. A postauricular incision was made and the muscles overlying the bulla were reflected posteriorly. A superior bullotomy was created with a microdrill to provide visual access to the round window. The cannula was passed through a smaller and inferiorly placed bullotomy into the bulla.
It was primed with the appropriate treatment solution and then moved slowly and carefully onto the round window niche under direct vision (as shown in Fig. 1). The cannula was fixed in position by applying a very small amount of adhesive to the external surface of the bulla. Durelon dental cement (3M ESPE, St Paul, MN, USA) was then applied to the external surface of the bulla, covering both the entry point of the cannula and the superior bullotomy. The cannula was fixed to the dorsal skull with Dacron mesh that had been passed extra-durally between two small holes drilled through the skull. The end of the cannula was then attached to the pump which was placed in a subcutaneous pocket made between the scapulae. The wound was closed with Vicryl sutures (Ethicon Inc., Somerville, NJ, USA) and aseptic staples (Leukoclip SD, Smith \& Nephew Pty Ltd, Clayton, VIC, Australia). Animals were administered the analgesic buprenorphine $(0.05 \mathrm{mg} / \mathrm{kg}$; Temgesic, Rechitt Benckiser, UK) to manage postoperative pain.

\section{Post-mortem assessment of cannula patency and histological methods}

At the end of the treatment period, all guinea pigs were euthanized with an overdose of sodium pentobarbitone (Lethabarb; Virbac Australia Pty. Ltd., Milperra, NSW, Australia), administered intraperitoneally, and perfused transcardially with a $0.9 \%$ saline solution followed by $4 \%$ paraformaldehyde (Mallinckrodt Baker Inc., Phillipsburg, NJ, USA).

To ensure pump patency, the tympanic membrane was viewed through the external auditory meatus with a surgical microscope and then incised to expose the round window membrane and tip of the cannula. The cannula was disconnected from the mini-osmotic pump and connected to a blunt 23-gauge needle attached to a saline-filled syringe. The cannula was deemed to be patent if saline was easily pushed through the cannula onto the round window.

After establishing cannula patency, the cochleae were extracted and placed in $10 \%(v / v)$ neutral-buffered formalin (formaldehyde $3.77 \% v / v$, phosphates as buffers, Fronine Laboratory Services, Cooper's Plains, QLD, Australia) for $24 \mathrm{~h}$. They were then decalcified over 14-21 days in 10\% ( $w / v)$ EDTA (EDTA Disodium Salt Dihydrate, Pronalys, Clayton, VIC, Australia) in $0.1 \mathrm{M}$ phosphate buffer. Cochleae were later embedded in OCT and sectioned in series on a cryostat in the paramodiolar plane with a section thickness of $12 \mu \mathrm{m}$ and mounted onto slides. The mounted sections were dehydrated in ethanol, stained with dilute acidified thionin, and cover-slipped with DPX (ProSciTech, Thuringowa, QLD, Australia). 
SGN density at each of the four cochlear turns was determined using five paramodiolar sections from each cochlea, with each section separated by $36 \mu \mathrm{m}$. All SGN soma with a visible nucleus within Rosenthal's canal were counted using bright-field microscopy at a magnification of $\times 400$. The area of Rosenthal's canal was measured at a magnification of $\times 100$ to convey SGN number as a density $\left(\mathrm{SGN} / \mathrm{mm}^{2}\right.$; NIH Scion Image analysis software). SGN density was calculated across each turn by averaging across all five cochlear sections. Density measures were corrected for possible overestimation using the method of Abercrombie (Coggeshall and Lekan 1996; Mouton 2002). For this, the diameter of the nucleus from SGNs across each cochlear turn and treatment group was measured. To achieve this, high-resolution images of each entire cochlea section were obtained using a Zeiss ICc1/ Marlin F146C model camera with a $\times 20$ magnification objective. The scanner was automatically calibrated to attain even Köhler illumination across all sections. All sections were scanned by a trained operator in one session. The resolution attained in each of the images was $0.32 \mu \mathrm{m} /$ pixel. A combination of Panoramic Viewer (version 1.14) and Nuclearquant software (version 1.14; 3D Histech, Budapest, Hungary) was used to determine nucleus size. Between 6 and 9 sections per animal across all 24 animals were used for these estimations. Within the software, the area of Rosenthal's canal was outlined, avoiding the intraganglionic spiral bundle for each cochlear turn. Only the lower side of each turn was analysed in order to expedite the analyses. Analysis of each slide was done blind as the slide label is automatically obscured by the software during analysis. A segmentation profile was established within the software with the parameters set as follows: radius range $=1.5-4 \mu \mathrm{m}$, minimum nuclei area $=7 \mu \mathrm{m}^{2}$, minimum circularity $=80$, smoothness $=5$, minimum contrast $=15$ and minimum intensity $=20$. This profile allowed automatic tracing of the perimeter of the nucleus within SGNs. These settings were liberal, selecting all nuclei and also selecting some aberrant data that were not nuclei. This liberal approach was chosen as the software later allowed the removal of unwanted data using a split image/gallery view. For each turn and treatment group, the SGN density was calculated and a correction for each was made using the calculated nucleus diameter for each corresponding turn and treatment group according to the formula: $N=$ $n[T /(T+D)]$, where $N=$ corrected estimate of SGNs, $n=$ uncorrected estimate, $T=$ thickness of the section $(12 \mu \mathrm{m})$ and $D=$ mean nucleus diameter $(\mu \mathrm{m})$. Although the corrections reduced the overall density estimations, they did not alter the differences seen between treatments and turns.

SGN somal area was measured in a similar way to that for nucleus diameter using the same scanned sections. The segmentation profile for detecting SGNs were: radius range $=3-12 \mu \mathrm{m}$, minimum nuclei area $=$ $10 \mu \mathrm{m}^{2}$, minimum circularity $=50$, smoothness $=1$, minimum contrast $=15$ and minimum intensity $=1$. These settings were again liberal and aberrant data were removed manually by the experimenter. Only SGNs that had a clear perimeter and a cytoplasm with a clear nucleus were selected for analysis. Somal area measurements from SGNs were averaged across sections to yield separate somal area estimations for each cochlear turn of each animal. Selected digital photomicrographs were taken of these scanned cochleae in the Panoramic Viewer software.

\section{Statistical analysis}

Statistical analyses were performed using SPSS Statistics for Windows version 19.0 (IBM Corp., Armonk, NY, USA). Analysis of variance (ANOVA) was used to test for significant differences in all dependent measures, with post hoc comparisons made using Bonferroni correction. Comparisons of ABR and DPOAE data across the repeated recording sessions (time) were made with repeated measures ANOVA, with time as a within-subjects variable. The length of surgery often precluded obtaining recordings immediately postsurgery; therefore, postsurgery data were not included in statistical analyses. For any instance of anacusis, the threshold was calculated as the highest intensity delivered (i.e. 92-97 dB SPL depending on the frequency). A difference between any factors was considered to be statistically significant if $p<0.05$.

\section{RESULTS}

This paper introduces a method of delivering neurotrophins to the cochlea in a relatively non-invasive and atraumatic manner using the cannula and miniosmotic pump system presented in Figure 1. The experiments performed in deafened animals were designed to demonstrate that BDNF was successfully delivered to the cochlea with the cannula by determining whether SGN density was greater when BDNF was administered than a saline control. The experiments in hearing animals used the same cannula system to determine the effect of BDNF upon ABRs, DPOAEs and SGN density.

\section{Cochlea histopathology}

Representative photomicrographs of cochlea sections from each experimental group are shown in Figures 2 and 3. The organ of Corti was degenerated in deafened animals, with typically no tunnel of Corti present and a flattened neuroepithelium. In normal 

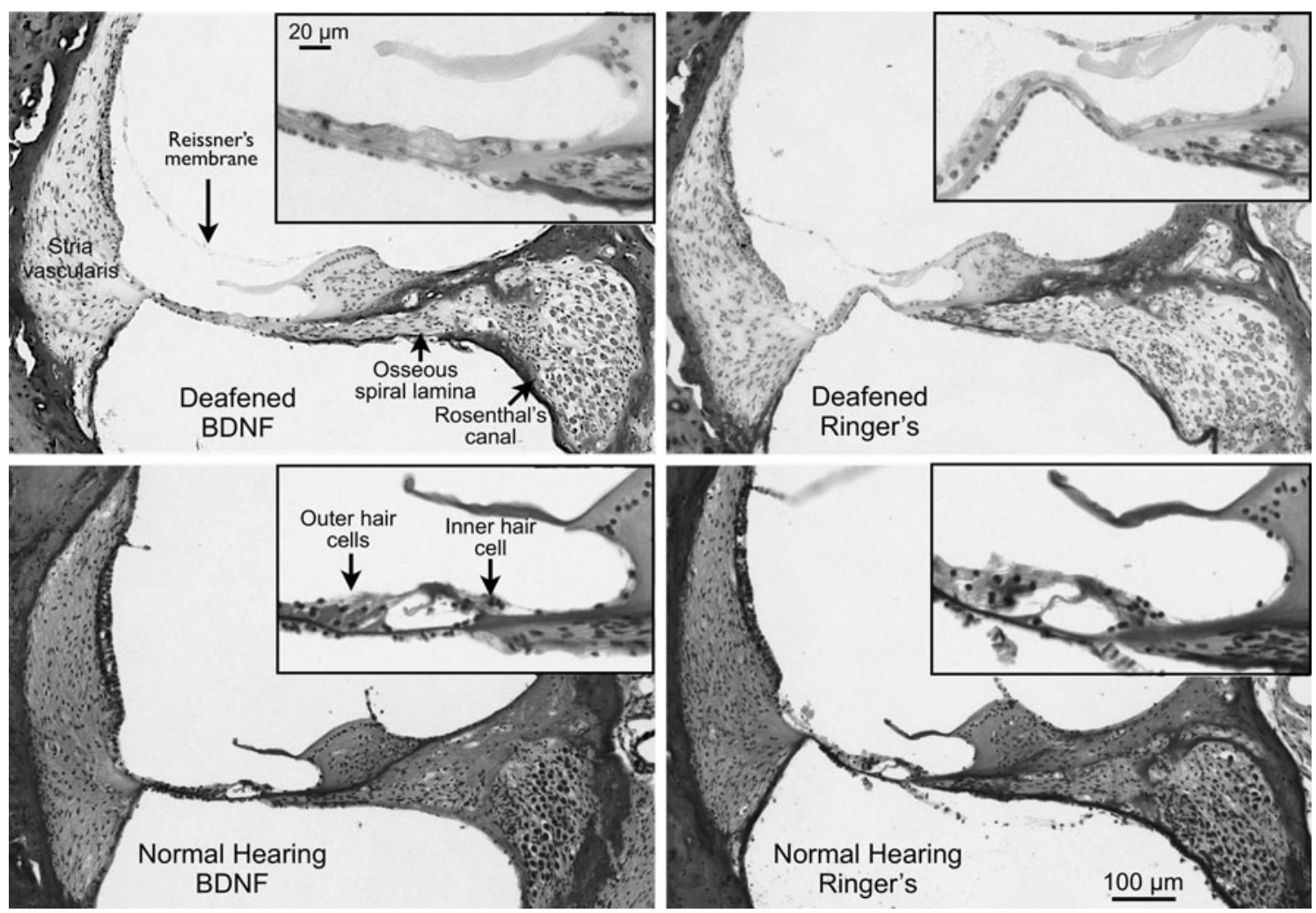

FIG. 2. Representative photomicrographs of the lower basal turn of the cochlea. Each image shows a representative section from one animal in each of the four experimental groups. Each inset shows higher-magnification images of the organ of Corti. In the deafened animals, the organ of Corti was degenerated and there were typically no remaining hair cells, while normal hearing animals treated with BDNF or Ringer's solution had intact inner and outer hair cells. In deafened animals, those treated with BDNF had greater preservation of peripheral dendrites in the osseous spiral lamina and SGNs in Rosenthal's canal, evidenced in the images by less white space in these regions. Normal hearing animals had the densest packing of neural tissue within the osseous spiral lamina and Rosenthal's canal, with no differences evident between those animals treated with either BDNF or Ringer's solution.

hearing animals, the organ of Corti appeared to be intact, with normal appearances of the tunnel and both the inner and the outer hair cells. BDNF treatment did not appear to alter the organ of Corti structure in either deafened or normal hearing animals compared to Ringer's-treated animals. The use of frozen cochlea sections in these studies precludes a more detailed examination of the organ of Corti integrity as this processing invariably causes damage to these structures. SGNs and neural processes within the osseous spiral lamina and Rosenthal's canal were more prevalent after BDNF treatment in deafened animals compared to Ringer's-treated animals. In both groups of deafened animals, Nissl staining often appeared dispersed within the cytoplasm, which may be an evidence of chromatolysis, while in both groups of normal hearing animals nuclear material appeared darker and more clearly defined.

\section{Spiral ganglion neuron density}

For deafened guinea pigs, SGN density in BDNFtreated animals was greater than that of Ringer'streated animals (Fig. 4a; ANOVA, main effect of treatment, $\left.F_{1,39}=9.23, p=0.004\right)$. The effect of BDNF was significantly different across cochlear turn (ANOVA, main effect of turn, $F_{3,39}=4.05, p=0.013$ ), with increased SGN density after BDNF treatment only observed in turns 1 and 2 of the cochlea (ANOVA, interaction of treatment and turn, $F_{3,39}=$ $4.65, p=0.007$ ). In turn 1 , the mean SGN density of BDNF-treated animals was $463 \pm 30$ (SEM) neurons/ $\mathrm{mm}^{2}$ compared to $227 \pm 44$ (SEM) neurons $/ \mathrm{mm}^{2}$ for Ringer's-treated animals. The effect of BDNF was smaller in turn 2, where mean SGN density for BDNFtreated animals was $354 \pm 18$ (SEM) neurons $/ \mathrm{mm}^{2}$ compared to $206 \pm 32$ (SEM) neurons $/ \mathrm{mm}^{2}$ in Ringer's-treated animals. This trophic support of SGNs in the basal region of the cochlea provided evidence that BDNF crossed the round window membrane with our delivery technique and therefore justified subsequent examination of the effects of BDNF treatment on the functional responses of normal hearing animals. Deafened animals treated with BDNF had 0.56 times lower SGN density in turn 1 and 0.42 times lower SGN density in turn 2 compared to normal hearing animals treated with BDNF (Fig. 4a). For normal hearing guinea pigs, SGN 

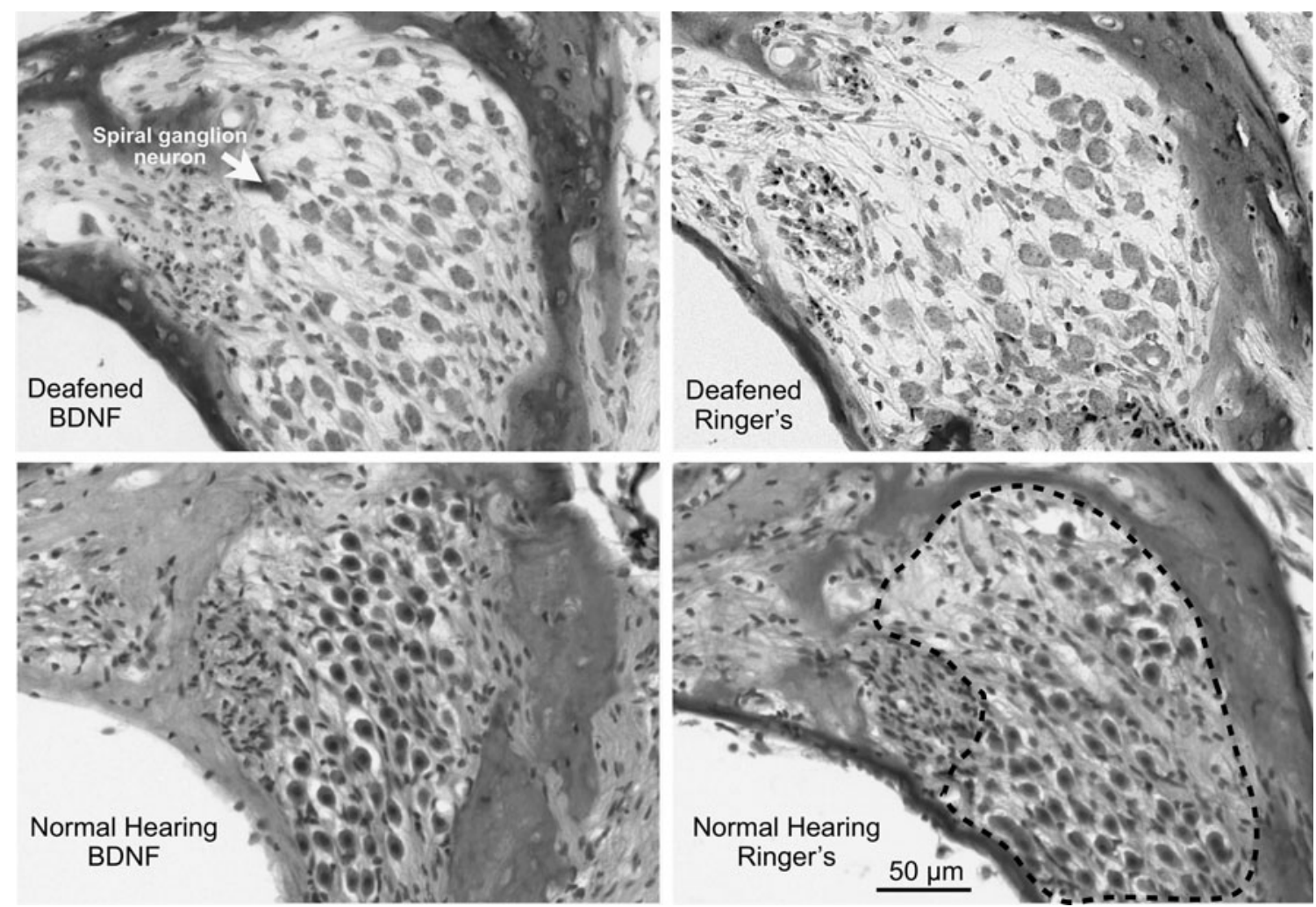

FIG. 3. Representative photomicrographs of SGNs in lower basal turn of the cochlea. These photomicrographs are higher-magnification images of those shown in Figure 2. The region of the Rosenthal's canal containing SGNs is outlined in the lower right section. In deafened animals, the density of SGNs was greater in animals treated with BDNF than those treated with Ringer's solution. In normal hearing animals, the density of SGNs was greater than that of the deafened animals and the SGNs showed less degeneration and more compact nuclei. The density of SGNs among normal hearing animals was similar in those treated with either BDNF or Ringer's solution.

density in BDNF-treated animals was not different to Ringer's-treated animals (ANOVA, main effect of treatment, $\left.F_{1,34}=0.12, p=0.91\right)$ in all turns of the cochlea.

\section{Spiral ganglion neuron somal area}

There was a significant effect of treatment group $\left(F_{3,74}=7.48, p<0.001\right)$ but not cochlear turn $\left(F_{3,74}=\right.$ $0.81, p=0.49$ ) upon SGN somal area (Fig. 4b). Post hoc testing revealed that soma were significantly larger in Ringer's-treated deafened animals than all other groups $(p<0.05$ on all comparisons). Somal size did not vary between any of the other groups (deafened BDNFtreated, normal hearing BDNF and Ringer's groups: $p=$ 1.0 on all comparisons with Bonferroni correction). We also examined the nucleus diameter within SGNs through a sample of 3,300 nuclei from all turns and sections across the 24 animals. There was a significant treatment effect $\left(F_{3}, 75=4.22, p=0.008\right)$ but no effect of turn $F_{3,75}=0.65, p=0.49$ ) and no interaction between treatment and turn. Post hoc tests revealed that the nucleus diameter of SGNs from deafened Ringer's-treated animals were significantly $(4.47 \%)$ smaller than those of deafened
BDNF-treated animals $(p=0.39)$; otherwise, all groups had similar SGN nucleus diameters.

\section{Auditory brainstem responses}

Example ABRs from normal hearing animals in response to acoustic clicks are shown in Figure 5. Wave III of the response was chosen for analysis of ABRs as this is the most robust wave in our recordings. The stimulus threshold, amplitude and latency of wave III of the ABRs were examined.

ABR thresholds in normal hearing animals in response to pure tones and clicks were examined at each recording time (Fig. 6). For pure tone stimuli, the ABR threshold varied across frequency, with a characteristic reduction in threshold between 4 and $16 \mathrm{kHz}$ (ANOVA, main effect of frequency, $F_{7}, 64=$ $36.28, p<0.001)$. ABR thresholds also varied across treatment (ANOVA, main effect of treatment, $\left.F_{1,64}=7.93, p=0.006\right)$ and time (ANOVA, main effect of time, $\left.F_{2}, 128=6.61, p=0.002\right)$ with significant interactions between both treatment and time (ANOVA, $F_{2}, 128=11.64, p<0.001$ ) and between treatment, time and frequency (ANOVA, $F_{14}, 128=$ $1.84, p=0.039)$. A transient increase in threshold was 

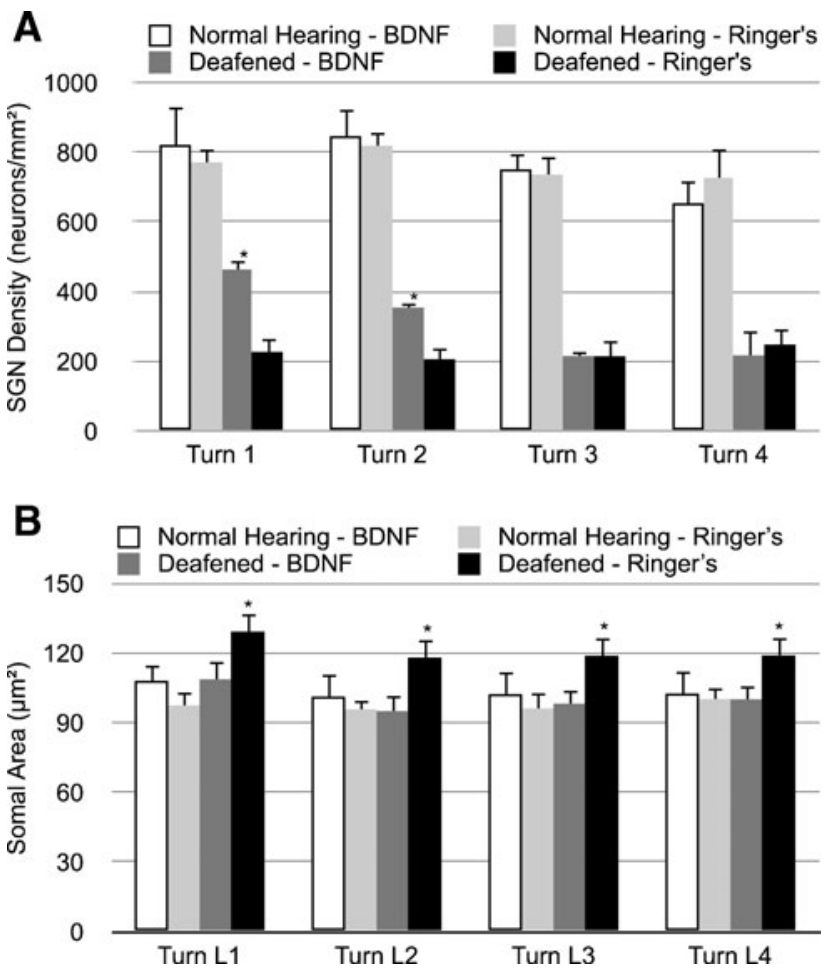

FIG. 4. The effect of treatment on SGN A density and B somal area. Each bar represents the mean \pm SEM density averaged across all animals in each group. In A asterisks represent groups significantly $(p<0.01)$ different from deafened Ringer's control group and in $\mathbf{B}$ asterisks represent groups significantly $(p<0.05)$ greater than all other groups. Five sections per animal were used for SGN density and between six and nine sections per animal were used for somal area estimations. Only the lower portions of cochlear turns were used for somal area estimations. The somal area sample was a relatively large sample of 7,470 soma across 24 animals. Deafened guinea pigs treated with BDNF through the round window membrane had a significantly greater preservation of SGNs in the lower turns of the cochlear spiral (numbered 1 and 2, turn 1 being the most basal) compared to deafened animals treated with Ringer's solution, suggesting that BDNF was successfully delivered across the intact round window membrane to the basal regions of the cochlea. Normal hearing guinea pigs treated with BDNF had similar SGN densities to normal hearing animals treated with Ringer's solution; the SGN density of each of these groups was considerably larger in turns 1 and 2 than the SGN density of deafened animals treated with BDNF. Deafened guinea pigs left treated with Ringer's solution had larger somal areas in all turns compared to all other groups. Normal hearing animals treated with BDNF had larger somal areas compared to normal hearing animals treated with Ringer's solution in turns $1-3$, with the greatest difference occurring in turn 1 .

observed immediately postsurgery for both treatment groups; however, at 1 week later, both groups appeared to have recovered, with thresholds in the BDNF group being lower than those in the Ringer's group at 8 and $16 \mathrm{kHz}$. After 4 weeks, the effect of treatment spanned the mid-high frequencies (2$32 \mathrm{kHz})$. At this time, ABR thresholds in the BDNF group at 16 and $32 \mathrm{kHz}$ were 35 and $28 \mathrm{~dB}$ SPL respectively lower than Ringer's animals. Furthermore, thresholds of BDNF-treated animals had dropped below pretreatment levels by an average of $11 \mathrm{~dB}$ SPL across $2-32 \mathrm{kHz}$ (Fig. 6). In contrast, thresholds in Ringer's-treated animals did not drop below pretreatment levels at any frequency and at some frequencies had not fully recovered 4 weeks posttreatment. In particular, at $32 \mathrm{kHz}$, nearly half of the Ringer's group had anacusis while every BDNF-treated animal could hear at this frequency. The responses to click stimuli (Fig. 6) across treatment and time were similar to those for pure tone stimuli, with a transient increase in threshold in both groups immediately postsurgery and with threshold for BDNF-treated animals progressively lower than those of Ringer's-treated animals after 1 and 4 weeks of treatment.

The amplitude of wave III in response to pure tone and click stimuli was also examined. ABR amplitude was plotted against stimulus intensity to generate $A B R$ growth functions at each recording time (Fig. 7). The aforementioned reduction in threshold in BDNF animals can also be observed in these functions as a shift of the curve to the left compared with Ringer's-treated animals. For statistical analyses of ABR amplitude, each individual growth function was normalized against its threshold to correct for this shift. To ensure equitable comparison, we only analysed amplitudes for ABR responses lying between threshold and $45 \mathrm{~dB}$ SPL above threshold for clicks and between threshold and $25 \mathrm{~dB}$ SPL above threshold for tones. Subsequently, all growth functions for both stimuli were generally monotonic and had similar trends across treatment groups; thus, data were analysed with repeated-measures ANOVA. For click-evoked ABR growth functions, there were main effects for treatment $\left(F_{1,108}=7.23, p=0.008\right)$ and time $\left(F_{2}, 107=8.08, p=0.001\right)$ and an interaction between treatment and time $\left(F_{2,107}=25.57, p<0.001\right)$. Post-hoc comparisons showed that across both treatment groups, ABR amplitudes were significantly reduced after 1 week $(p<0.001)$ and 4 weeks $(p<0.001)$ compared to pretreatment. The reduction in amplitude growth 1 week after surgery was of similar magnitude for both BDNF and Ringer's groups $\left(F_{1,108}=0.47, p=0.494\right)$. Four weeks after surgery, the click amplitudes of Ringer's-treated animals had dropped even further, but growth amplitudes in the BDNF group were significantly greater $\left(F_{1,115}=36.63, p<0.001\right)$ and had grown to exceed pretreatment levels for most stimulus intensities less than $60 \mathrm{~dB}$ SPL. Above this stimulus level, the response reached its plateau. This difference appeared to be due to the reduction in the rate of click amplitude growth in Ringer's-treated animals as there was not a significant change in click amplitude growth over time in BDNFtreated animals.

Similar results for ABR growth function were observed in response to high frequency pure tones 


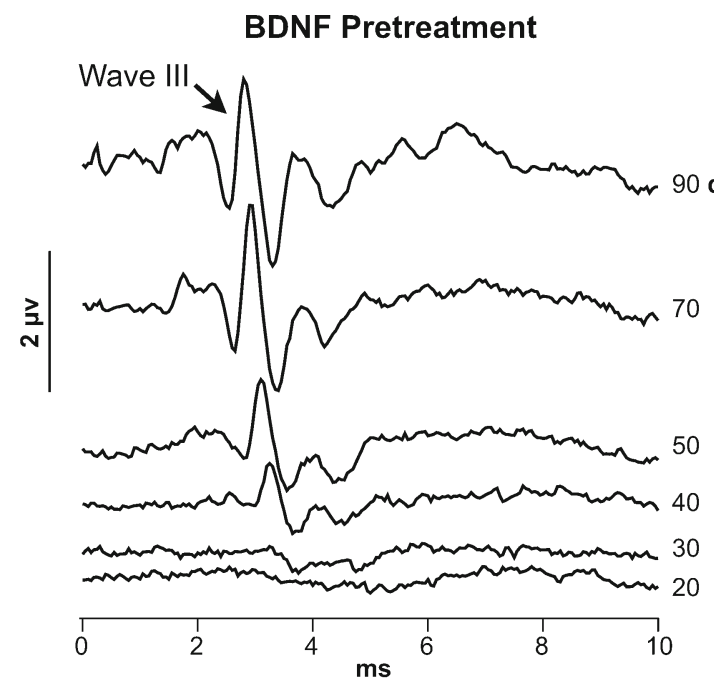

Ringers Pretreatment

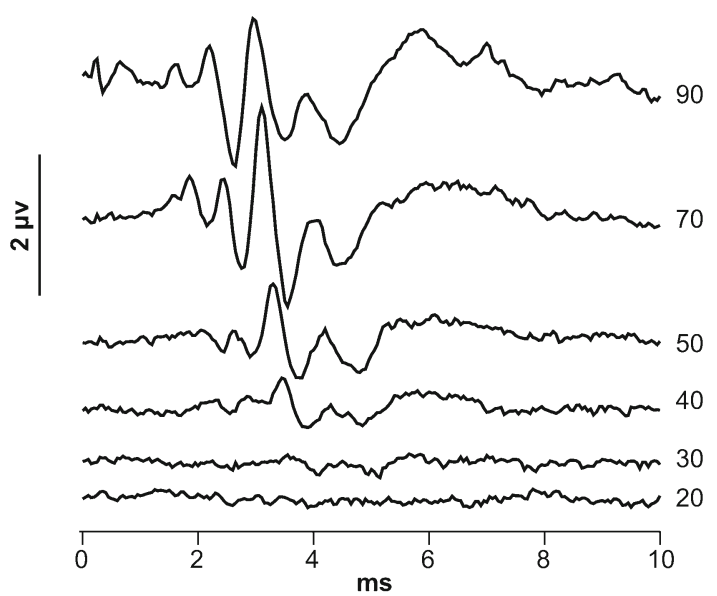

BDNF 4 Weeks

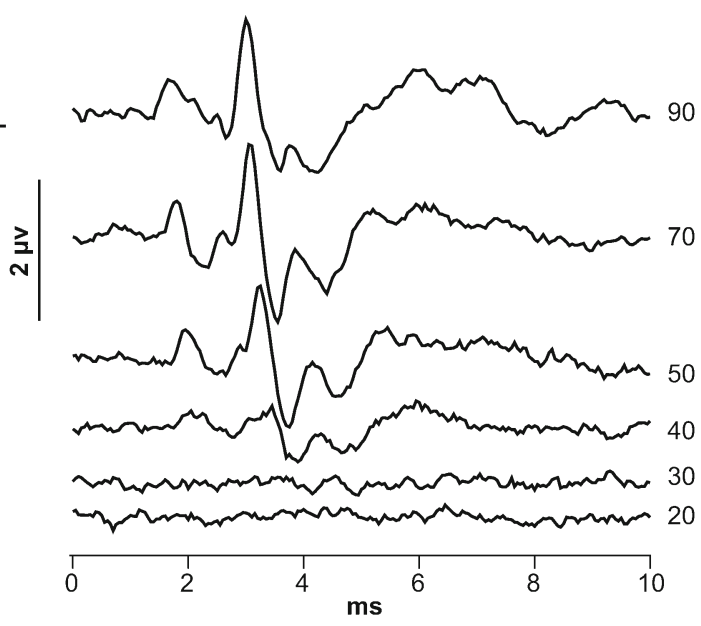

Ringers 4 Weeks

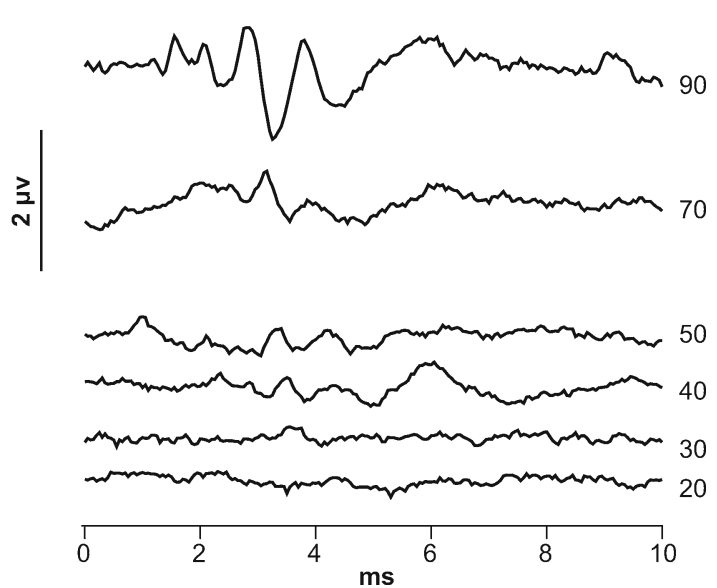

FIG. 5. Example ABR responses to acoustic click stimulation in normal hearing animals. Prior to treatment, the amplitude of wave III of the ABR response was similar in animals within the BDNF or Ringer's group. By 4 weeks posttreatment, the amplitude and threshold of wave III had reduced in animals treated with Ringer's solution, but in BDNF-treated animals the response remained similar to that prior to treatment. While there was a characteristic reduction in the latency (time of occurrence) of wave III with increasing stimulus amplitude, there was no difference in the latency of this wave among the groups.

(Fig. 7). Amplitude growth functions for pure tones at 16 and $32 \mathrm{kHz}$ resembled the click-evoked data. In all animals, there was a reduction in amplitude growth 1 week after surgery which progressively worsened in Ringer's-treated animals but recovered to exceed pretreatment levels in the BDNF group. At $8 \mathrm{kHz}$, the deterioration of amplitude growth over time in the Ringer's group was less marked, but the trophic BDNF effect was still apparent. The growth functions did not differ between the treatment groups, or across time, at $2 \mathrm{kHz}$, but at $4 \mathrm{kHz}$ BDNF amplitudes were slightly greater than those in the Ringer's group. Below $2 \mathrm{kHz}$, again the Ringer's-treated group had significantly lower amplitudes at 4 weeks compared to the BDNF group.

To determine the effect of BDNF treatment upon temporal aspects of the $\mathrm{ABR}$, response latencies above the threshold were examined. Clicks were chosen for this analysis because the transient nature of the stimulus compared to pure tones reduced the temporal dispersion of the ABR peaks. Wave III latencies were similar across the groups prior to surgery. In both BDNF- and Ringer's-treated animals, latencies were prolonged immediately after surgery and returned close to pretreatment times by 1 week after surgery. However, there were no significant differences between the treatment groups.

\section{Distortion product otoacoustic emissions}

DPOAE amplitudes were analysed in response to both 65 and $75 \mathrm{~dB}$ SPL stimulation (data for $65 \mathrm{~dB}$ SPL shown in Fig. 8). Responses to 45 and $55 \mathrm{~dB}$ SPL stimulation were also recorded, but these were 

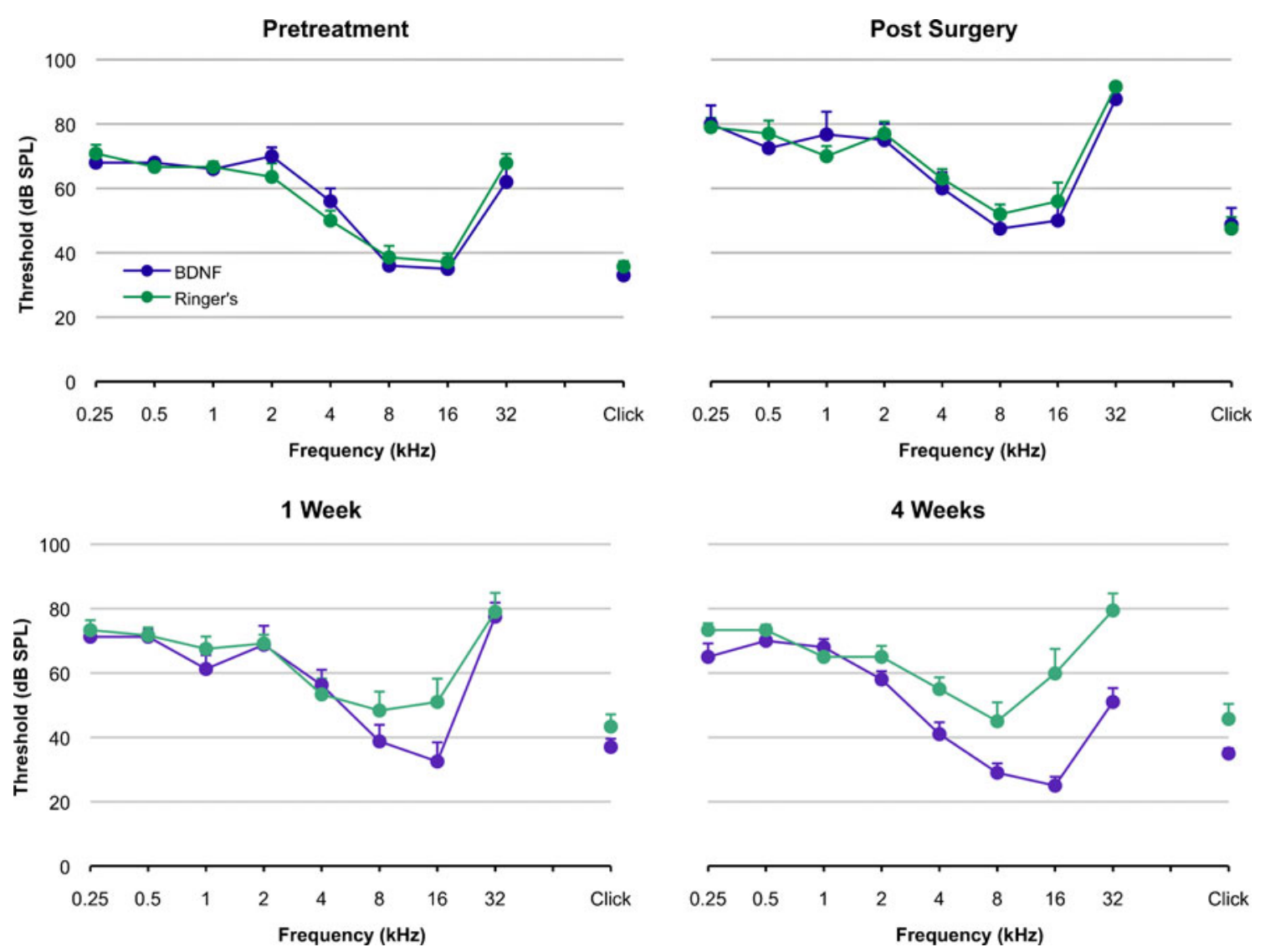

FIG. 6. The effect of treatment on ABR threshold. Each data point represents the mean \pm SEM threshold across animals. Note that the data for clicks are presented as individual points on the right side of each graph. Prior to implant, there was little difference between treatments, with both BDNF- and Ringer's-treated animals having the lowest thresholds between 4 and $16 \mathrm{kHz}$. Immediately post-surgery, the thresholds in both groups had risen by $5-10 \mathrm{~dB}$ SPL across most stimulus frequencies, including clicks. After 1 week, the thresholds of both groups had dropped, although those for BDNF had dropped more. After 4 weeks, the threshold in the Ringer's group had stabilized, being similar to those at 1 week, but not fully recovering to pretreatment levels in the mid-high frequencies $(4-16 \mathrm{kHz})$. However, after 4 weeks, the thresholds for the BDNF group dropped further to the point where they were below pretreatment levels and up to $29 \mathrm{~dB}$ SPL lower than the Ringer's group in the mid-high frequencies $(2-32 \mathrm{kHz})$. Note that the data recorded immediately post-surgery were not included in statistical analyses as sufficient data were not recorded in all animals.

found to fall below the noise floor. As expected for DPOAE recordings, amplitudes were greater in response to 75 compared to $65 \mathrm{~dB}$ SPL stimulation and the responses varied across stimulus frequency. Notably, DPOAE amplitudes reduced over time across most frequencies (ANOVA main effect of time, $\left.F_{2,284}=35.62, p<0.001\right)$. The average reduction by $5 \mathrm{~dB}$ SPL was significant between pretreatment and at 1 week $\left(F_{1,285}=41.52, p<0.001\right)$, but there was no change in the DPOAE amplitude between 1 and 4 weeks $\left(F_{1}, 323=1.01, p=0.32\right)$. Although there was a main effect of treatment $\left(F_{1}, 285=9.37, p=0.002\right)$, this was due to the lower amplitudes at pretreatment in the Ringer's compared to the BDNF group, and this pattern remained across the duration of treatment. There were no significant interactions between treatment and time $\left(F_{2,284}=2.03, p=0.133\right)$ or treatment and any other variable, suggesting that treatment had no effect on DPOAE amplitude.

\section{DISCUSSION}

In this study, the improved survival of SGNs with a cannula delivery system in deafened animals is evidence for its efficacy at delivering BDNF to the lower two cochlear turns through the round window. This is consistent with studies demonstrating that BDNF (Endo et al. 2005; Havenith et al. 2011) and NT-3 (Noushi et al. 2005) are capable of crossing the round window membrane and effecting improved SGN survival across the lower turns of the deafened guinea pig cochlea. The lack of effective delivery to the upper cochlear turns was expected given that tracer studies demonstrate that substances placed on the round window membrane do not disperse equally through the cochlea, instead appearing in minimal amounts in more apical regions with higher concentrations in the basal turns (Saijo and Kimura 1984). In addition, NT-3 administered through a basal turn cochleostomy produces much higher concentrations 
in the lower turns of the cochlea (Richardson et al. 2004). With respect to SGN survival, the round window delivery method used here rescued approximately a third of the neurons seen in normal hearing animals. It was therefore more effective than neurotrophin delivery from a polymer but less effective than delivery via an intracochlear cannula. However, this approach had the distinct advantage of interfering much less with hearing.

Unlike previous studies in deafened guinea pigs (Shepherd et al. 2005; Agterberg et al. 2008), rats (McGuinness and Shepherd 2005) and cats (Leake et al. 2011), we did not show an increase in SGN somal area after BDNF treatment, which may be due to a reduced amount of BDNF reaching the SGNs. Delivery of BDNF to the intact round window membrane of the guinea pig via either gelfoam (Havenith et al. 2011) or cannula as in the present studies did not promote SGN survival as much as that seen when BDNF is delivered directly into the scala tympani (McGuinness and Shepherd 2005; Shepherd et al. 2005; Agterberg et al. 2008; Leake et al. 2011), suggesting that the amount of $\mathrm{BDNF}$ reaching the SGNs is reduced via round window administration. If so, the amount of BDNF absorbed across the round window and distributed within the scalae and tissues may be sufficient to promote SGN survival but not to increase SGN somal area.

The increased somal size that we observed in deafened Ringer's-treated animals matches the observations of Dodson and Mohuiddin (2000), who showed that aminoglycoside-deafened guinea pigs showed progressively increased SGN somal areas at 1, 3 and 6 weeks (up to $34 \%$ larger) after deafening with a corresponding decrease in myelination compared to
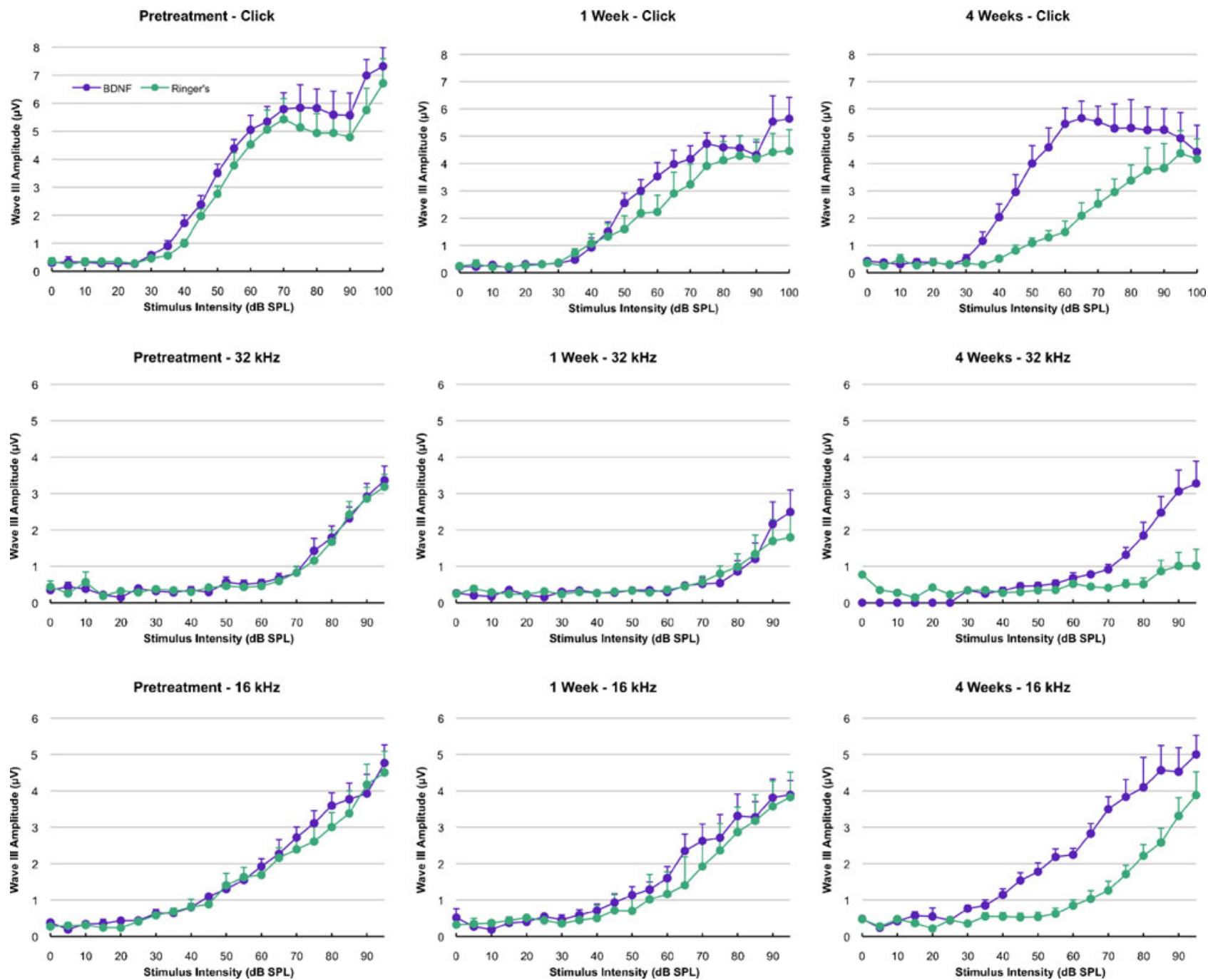

FIG. 7. The effect of treatment on ABR amplitude. Each data point represents the mean \pm SEM of the amplitude of wave III of the auditory brainstem response. Prior to implant, there were no differences in the amplitude growth function between the BDNF and Ringer's treatment groups. The rising function of the ABR amplitude with increasing stimulus intensity was similar across all the pure tone frequencies; the response to clicks starts at lower intensities and rises to greater amplitudes, reflecting the broader excitation of the click stimulus across the cochlea (note the different vertical axis scales for the click and tone pip data). After 4 weeks of treatment, the ABR amplitudes in BDNF-treated animals exceeded pretreatment levels. However, over the same period, the ABR amplitudes in the Ringer's group had dropped across most frequencies. 

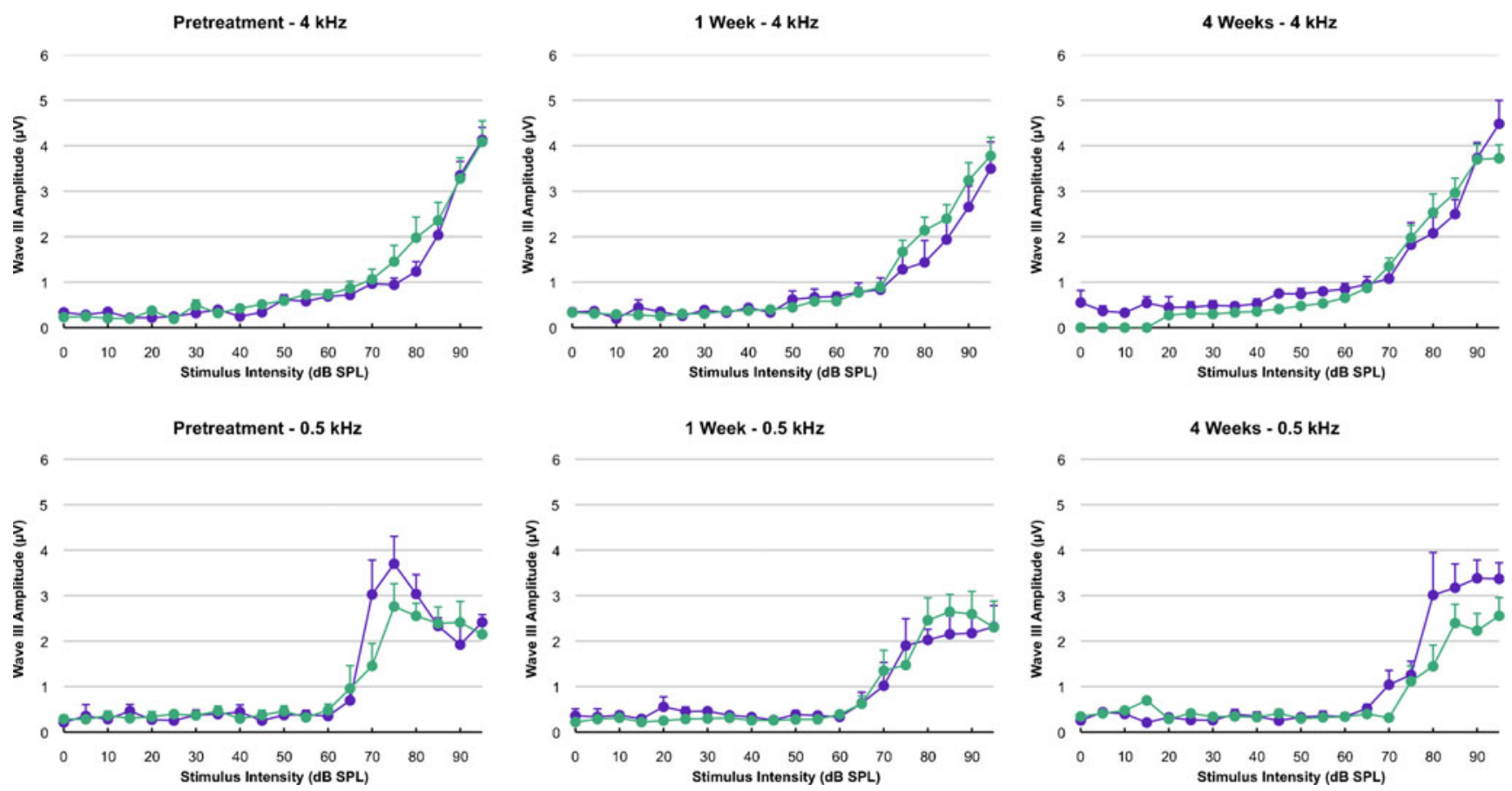

FIG. 7. (continued)

controls. They attribute the increased size to an increase in protein synthesis after response injury, possibly due to an increase in endogenous neurotrophins released from central targets. Other studies in mammals showing a decrease in SGN somal area after aminoglycoside deafening (Leake and Hradek 1988; Nadol et al. 1989; Shepherd et al. 2005) have in general examined longer periods of survival after deafening where Dodson has similarly shown a reduction in SGN size.

In hearing animals, a 4 week treatment with BDNF resulted in significantly decreased tone and click ABR thresholds. By 1 week of treatment, thresholds in the BDNF group had dropped below pretreatment levels at $16 \mathrm{kHz}$, and this trend continued so that by 4 weeks thresholds had dropped below pretreatment levels across 4-32 kHz. The threshold effects were derived from the lower two cochlear turns, as predicted by Greenwood (1990), which was the same region shown to receive trophic support in deafened cochleae (Fig. 4a). There are therefore reasonable grounds to consider this functional effect to be a consequence of BDNF treatment. These findings are consistent with the findings of studies by Meen and colleagues, where a single dose of $0.5 \mu \mathrm{g}$ BDNF was infused into the cochlea 1 month after cisplatin toxicity. In their first study, a significant protective effect of BDNF was found on ABR thresholds to tones for the next 3 months, but there was also a significant variance in the data due to anacusis in many animals (Meen et al. 2009). The protection provided by BDNF was not found in response to clicks in a subsequent study (Meen et al. 2010). This may not be surprising given that here we have found that the effects of BDNF are greater at high frequencies compared to clicks, possibly reflective of the fact that most spectral energy with our click stimulus is below $8 \mathrm{kHz}$. Several other methodological approaches in the studies of Meen and colleagues may have weakened any protective effect afforded by BDNF, including the intracochlear delivery, the single application and the removal of half of the tympanic membrane to access the cochlea.

Recent studies by Havenith et al. (2011) showed that BDNF delivered via gelfoam to the round window membrane of deafened guinea pigs did not result in any change in threshold, latency or amplitude of an electrically evoked ABR response. They also showed a similar degree of SGN survival to our studies across turns 1 and 2 of the cochlea, with no increase in somal area. As suggested above, we propose that their findings of no effect on function may be due to the reduced concentration of drug delivered to SGNs.

The present study is the first to demonstrate that the auditory system is responsive to the effects of neurotrophins even in cochleae that are close to normal hearing, as has been achieved with the cannula-based delivery method used here. This is an important observation given that some other trophic effects of neurotrophins, such as the propensity to regrow or extend peripheral dendrites, are seen only in damaged areas of the organ of Corti of deafened cochleae (Wise et al. 2005, 2010). However, the effects 

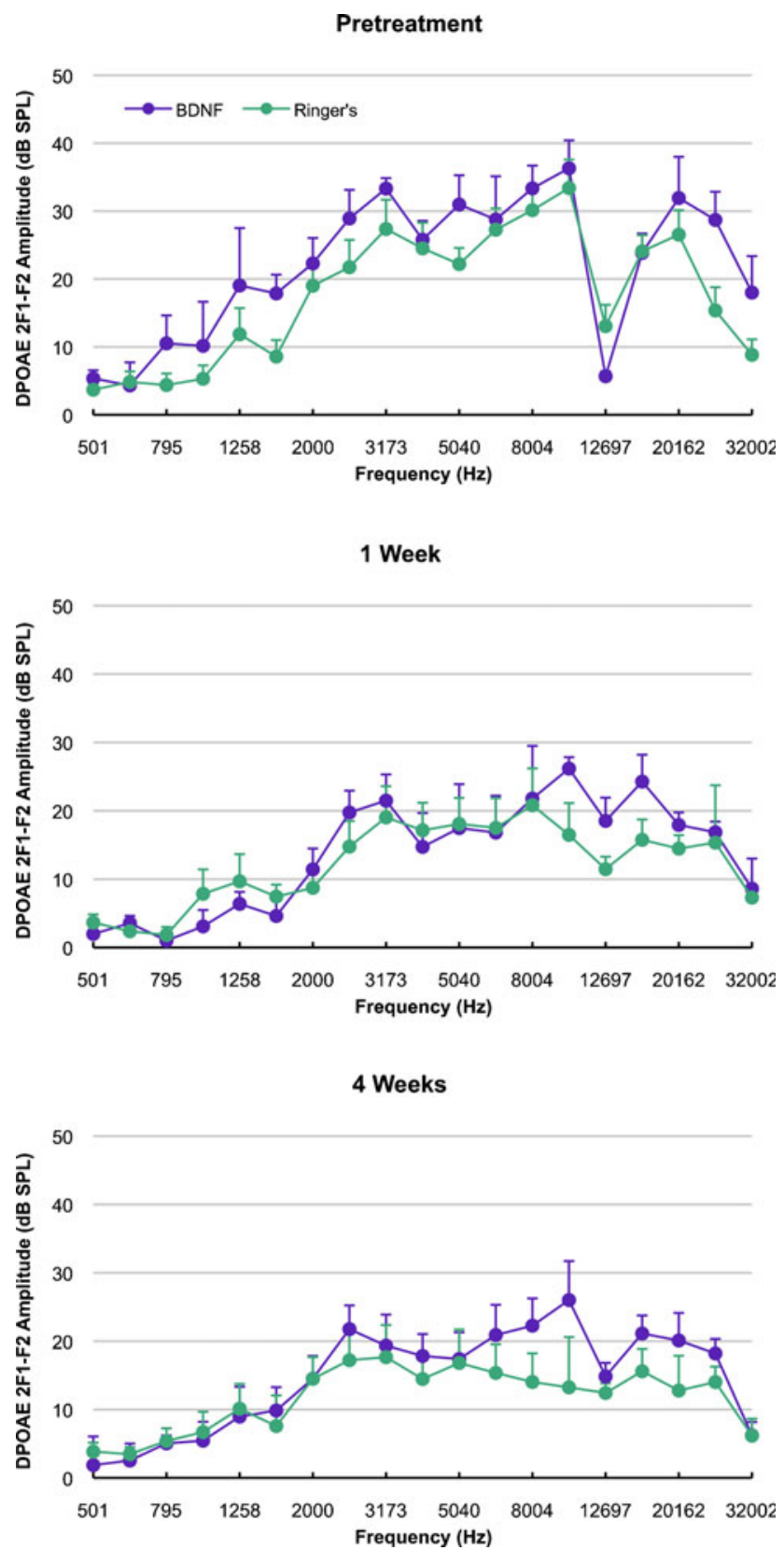

FIG. 8. The effect of treatment on DPOAE amplitude. Each point represents the mean \pm SEM DPOAE amplitude in response to a 65 - $d B$ $\mathrm{SPL}$ stimulus. The amplitude is the $2 \mathrm{~F} 1-\mathrm{F} 2$ distortion product, which was typically the largest distortion product response. The frequency is the geometric mean of F1 and F2 stimulus frequencies. Prior to treatment, the DPOAE response amplitudes were slightly larger in the BDNF group. No significant differences were found between treatments. There was a drop in DPOAE amplitude across most frequencies in both groups by 1 week that stabilized by 4 weeks. The diminished responses seen at 12 and $32 \mathrm{kHz}$ in most of the data reflect our observations that DPOAEs were regularly lower at these frequencies in each guinea pig.

observed may have been anticipated given known effects of neurotrophins upon neurons; neurotrophins are known to regulate ion channel expression and to modulate firing patterns and neuronal excitability throughout the central nervous system (Holm et al. 1997; Lesser et al. 1997; Kafitz et al. 1999; Rose et al. 2004; Davis-Lopez de Carrizosa et al. 2009; Luther and Birren 2009). Furthermore, it is acknowledged that the method of delivery of neurotrophins, while minimally invasive, likely caused a degree of cochlear dysfunction as evidenced by the reduction in DPOAE amplitudes, as discussed in the following paragraph. Whether this may have influenced the effect of BDNF upon auditory thresholds is beyond the scope of the present study.

There was no evidence for the threshold reduction being mediated at the level of the outer hair cells. DPOAE amplitudes were reduced to a similar extent in both BDNF- and Ringer's-treated ears, with the effect stabilizing after the first week of treatment. This argues against a specific effect of either treatment given that the DPOAEs remained unchanged for the last 3 weeks of treatment and argues in favour of cochlear trauma related to the implantation of the cannula. This is plausible given that bone drilling to open the bulla for cannula placement may have caused limited acoustic trauma (Pau et al. 2007) as may the placement of the cannula over the round window. Furthermore, the presence of the cannula may have led to a change in round window membrane compliance with damping of cochlear mechanics, and this may have reduced DPOAE amplitudes. Finally, DPOAE amplitudes decreased, although to a lesser extent, in the contralateral (unimplanted) ear, leaving the possibility that systemic factors, such as surgical stress, may also have contributed to a reduction in outer hair cell function bilaterally. Recent evidence suggests that this is unlikely to be an effect of crossed auditory pathways (Larsen and Liberman 2010).

The effects of BDNF in improving ABR thresholds and amplitude growth occurred between 1 and 4 weeks after surgery, when DPOAE responses had stabilized. Over the same period of time, amplitude growth in the Ringer's-treated animals continued to deteriorate. This argues for a BDNF treatment effect at the level of the auditory nerve. We postulate that the threshold of ABRs to acoustic stimulation improved through a direct trophic effect upon the auditory nerve and/or its synapse with the inner hair cells. Electrically evoked ABR thresholds have been shown to be reduced below pretreatment levels in deafened animals treated with BDNF (Shinohara et al. 2002; Yamagata et al. 2004; Shepherd et al. 2005; Leake et al. 2011). It therefore seems possible that treatment with neurotrophins may affect the auditory neurons in the hearing cochlea to a similar degree as the deafened cochlea. While details of the mechanisms for this are not yet known, BDNF treatment has several effects upon neural excitability that might contribute to the observed effects. The direct application of BDNF to cortex, cerebellar or hippocampal cells 
increased neuronal excitability via a Trk-dependent activation of a sodium ion conductance (Kafitz et al. 1999). NT-3 has an excitatory effect upon both the spontaneous and glutamate-evoked firing rates in the peripheral dendrites of auditory neurons during iontophoresis of the cochlea. While these studies and others (Lesser et al. 1997; Rose et al. 2004; Davis-Lopez de Carrizosa et al. 2009; Luther and Birren 2009) reveal that neurotrophins can increase neuronal excitability, the effects occur extremely rapidly, within milliseconds of application, and alternate mechanisms may be required to explain the effects which emerged after several weeks of BDNF application.

ABR growth functions from clicks and high-frequency tones continued to deteriorate in Ringer's-treated animals over a month after surgery, even though the DPOAE amplitudes stabilized after the first postoperative week. These findings argue strongly for cochlear trauma having been caused by implantation of the cannula. The pattern of response bears some resemblance to the effects of noise trauma on amplitude growth observed by Kujawa and Liberman (2006, 2009). These investigators found that excitotoxicity caused by noise exposure disrupted synapses between the inner hair cells and peripheral axons of the auditory nerve. The majority of these synapses did not recover, leading to a reduction in ABR amplitude growth, and later to a loss of SGNs (Kujawa and Liberman 2009). We also found a reduction in amplitude growth in Ringer's-treated animals but this deteriorated gradually over a month, unlike in Kujawa and Liberman where the loss was apparently early and remained relatively stable. Without an assessment of synaptic ribbons, it is not possible to know whether cannula implantation and Ringer's administration lead to dysfunction of the organ of Corti by excitotoxicity, but whatever the mechanism it would appear that BDNF protected against this effect because ABR growth amplitudes recovered or improved to exceed normal levels. It is worth noting that neurotrophins assist the regeneration of synaptic ribbons after excitotoxicity in vitro (Wang and Green 2011), and a similar effect may occur after acoustic trauma in vivo as there are several reports in the literature that reveal a protective effect of neurotrophins upon acoustic noise trauma (Shoji et al. 2000; Diao et al. 2007).

The present experiment raises the possibility that neurotrophins may have a role in maintaining or improving acoustic hearing following cochlear implantation. Whether this will require continuous delivery or an interval of treatment remains to be determined and will depend on a better understanding of the mechanism for neurotrophins protecting the inner ear. However, the present experiment demonstrates that the potential role of neurotrophins in cochlear implantation include hearing protection as well as protection of the auditory nerve. Delivery methods for neurotrophins of potential clinical significance include elution from a polymer coating the cochlear electrode (Richardson et al. 2009), round window delivery (Noushi et al. 2005) or release from genetically modified cells (Pettingill et al. 2008). Our results suggest that round window delivery will only be effective in improving higher frequencies that arise from the basal turns of the cochlea, so its utility may be limited to individuals with functional low-frequency hearing where BDNF could be applied in an attempt to improve high-frequency thresholds. Prior to clinical application, the effects of BDNF upon the phenotypic and ion channel expression of SGNs will also need to be examined. When BDNF is applied to cultures of early post-natal auditory neurons (Adamson et al. 2002; FloresOtero et al. 2007), apical SGNs behave more like basal neurons, with NT-3 having the opposite effect. Whether neurotrophins have similar effects upon SGNs in the mature animal is unknown, but if so this may influence the survival characteristics, function and/or choice of preferred trophic factor for clinical application.

The improvement in ABR thresholds observed in this study also leaves open the possibility that BDNF may improve hearing following sensorineural hearing loss. These results could influence the interpretation of recent clinical trials where insulin-like growth factor-1 was applied to the round window via a polymer and improved hearing more frequently than historical controls in patients with sudden sensorineural hearing loss (Nakagawa et al. 2010). It may be that insulin-like growth factor-1 is reducing thresholds within the auditory system rather than repairing or protecting a damaged organ of Corti.

\section{ACKNOWLEDGEMENTS}

We thank Ms. Helen Feng for electrode manufacturing, Ms. Elisa Borg for animal husbandry assistance, Mr. Frank Nielsen for engineering assistance, Mrs. Maria Clarke and Ms. Prue Nielsen for assistance with histology, Ms. Niles Nelson and Ms. Elizabeth Manning for general assistance, Dr. James Fallon for ABR analysis software, and Dr. Justin Tan and anonymous reviewers from the Journal for their constructive comments on the manuscript. Funding for this work was provided by the National Health and Medical Research Council of Australia and the Royal Victorian Eye and Ear Hospital.

\section{REFERENCES}

Adamson CL, ReID MA, Davis RL (2002) Opposite actions of brainderived neurotrophic factor and neurotrophin-3 on firing features and ion channel composition of murine spiral ganglion neurons. J Neurosci 22:1385-1396 
Agterberg MJ, Versnel H, de Groot JC, Smoorenburg GF, Albers FW, KLIS SF (2008) Morphological changes in spiral ganglion cells after intracochlear application of brain-derived neurotrophic factor in deafened guinea pigs. Hear Res 244:25-34

Coggeshall RE, LeKan HA (1996) Methods for determining numbers of cells and synapses: a case for more uniform standards of review. J Comp Neurol 364:6-15 (Erratum in 369:162)

Davis-Lopez de Carrizosa MA, Morado-Diaz CJ, Tena JJ, BenitezTemino B, Pecero ML, Morcuende SR, de la Cruz RR, Pastor AM (2009) Complementary actions of BDNF and neurotrophin-3 on the firing patterns and synaptic composition of motoneurons. J Neurosci 29:575-587

Diao MF, Gao WY, Sun JJ, Liu Y, Chen DL, Jiang W, Zhao J, Chen X (2007) Protection from noise-induced hearing loss by a nitric oxide synthase inhibitor and neurotrophin 3 in the guinea pig cochlea. Zhonghua Er Bi Yan Hou Tou Jing Wai Ke Za Zhi 42:281-285

Dodson HC, Mohuiddin A (2000) Response of spiral ganglion neurones to cochlear hair cell destruction in the guinea pig. J Neurocyt 29:525-537

Endo T, Nakagawa T, Kita T, Iguchi F, Kim TS, Tamura T, Imai K, TаватA Y, Iто J (2005) Novel strategy for treatment of inner ears using a biodegradable gel. Laryngoscope 115:2016-2020

Evans AJ, Thompson BC, Wallace GG, Millard R, O’Leary SJ, Clark GM, Shepherd RK, Richardson RT (2009) Promoting neurite outgrowth from spiral ganglion neuron explants using polypyrrole/BDNF-coated electrodes. J Biomed Mater Res A 91:241-250

Flores-Otero J, Xue HZ, Davis RL (2007) Reciprocal regulation of presynaptic and postsynaptic proteins in bipolar spiral ganglion neurons by neurotrophins. J Neurosci 27:14023-14034

Gantz BJ, Turner C, Gfeller KE, Lowder MW (2005) Preservation of hearing in cochlear implant surgery: advantages of combined electrical and acoustical speech processing. Laryngoscope 115:796-802

Gillespie LN, Clark GM, Bartlett PF, Marzella PL (2003) BDNFinduced survival of auditory neurons in vivo: cessation of treatment leads to accelerated loss of survival effects. J Neurosci Res 71:785-790

GillesPie LN, Clark GM, Marzella PL (2004) Delayed neurotrophin treatment supports auditory neuron survival in deaf guinea pigs. Neuroreport 15:1121-1125

GREENWOOD DD (1990) A cochlear frequency-position function for several species-29 years later. J Acoust Soc Am 87:2592-2605

Havenith S, Versnel H, Agterberg MJ, de Groot JC, Sedee RJ, Grolman W, KLIS SF (2011) Spiral ganglion cell survival after round window membrane application of brain-derived neurotrophic factor using gelfoam as carrier. Hear Res 272:168-177

Heffer LF, Sty DJ, Fallon JB, White MW, Shepherd RK, O’Leary SJ (2010) Examining the auditory nerve fiber response to high rate cochlear implant stimulation: chronic sensorineural hearing loss and facilitation. J Neurophysiol 104:3124-3135

Hellier WP, Wagstaff SA, O'Leary SJ, Shepherd RK (2002) Functional and morphological response of the stria vascularis following a sensorineural hearing loss. Hear Res 172:127-136

Holm NR, Christophersen P, Olesen SP, Gammeltoft S (1997) Activation of calcium-dependent potassium channels in mouse [correction of rat] brain neurons by neurotrophin-3 and nerve growth factor. Proc Natl Acad Sci U S A 94:1002-1006

Kafitz KW, Rose CR, Thoenen H, Konnerth A (1999) Neurotrophinevoked rapid excitation through TrkB receptors. Nature 401:918-921

KuJAWA SG, Liberman MC (2006) Acceleration of age-related hearing loss by early noise exposure: evidence of a misspent youth. J Neurosci 26:2115-2123

KuJAWA SG, Liberman MC (2009) Adding insult to injury: cochlear nerve degeneration after "temporary" noise-induced hearing loss. J Neurosci 29:14077-14085
Larsen E, Liberman MC (2010) Contralateral cochlear effects of ipsilateral damage: no evidence for interaural coupling. Hear Res 260:70-80

Leake PA, Hradek GT (1988) Cochlear pathology of long term neomycin induced deafness in cats. Hear Res 33:11-33

Leake PA, Hradek GT, Hetherington AM, Stakhovskaya O (2011) Brain-derived neurotrophic factor promotes cochlear spiral ganglion cell survival and function in deafened, developing cats. J Comp Neurol 519:1526-1545

LEsSER SS, SHERWOOD NT, Lo DC (1997) Neurotrophins differentially regulate voltage-gated ion channels. Mol Cell Neurosci 10:173183

Luther JA, Birren SJ (2009) p75 and TrkA signaling regulates sympathetic neuronal firing patterns via differential modulation of voltage-gated currents. J Neurosci 29:5411-5424

McGuinness SL, SHEPHERd RK (2005) Exogenous BDNF rescues rat spiral ganglion neurons in vivo. Otol Neurotol: Official publication of the American Otological Society, American Neurotology Society [and] European Academy of Otology and Neurotology 26:1064-1072

Meen E, Blakley B, Quddusi T (2009) Brain-derived nerve growth factor in the treatment of sensorineural hearing loss. Laryngoscope 119:1590-1593

Meen E, Blakley B, Quddusi T (2010) Does intracochlear brainderived nerve growth factor improve auditory brainstem click thresholds in sensorineural hearing loss? J Otolaryngol Head Neck Surg 39:232-235

Miller JM, Chi DH, O'Keeffe LJ, Kruszka P, Raphael Y, Altschuler RA (1997) Neurotrophins can enhance spiral ganglion cell survival after inner hair cell loss. Int J Dev Neurosci 15:631-643

Miller JM, Le Prell CG, Prieskorn DM, Wys NL, Altschuler RA (2007) Delayed neurotrophin treatment following deafness rescues spiral ganglion cells from death and promotes regrowth of auditory nerve peripheral processes: effects of brain-derived neurotrophic factor and fibroblast growth factor. J Neurosci Res 85:1959-1969

Mouton PR (2002) Principles and practices of unbiased stereology. Johns Hopkins University Press, Baltimore

NADOL JB, Young YS, GLYNN RJ (1989) Survival of spiral ganglion cells in profound sensorineural hearing loss: implications for cochlear implantation. Ann Otol Rhinol Laryngol 98:411-416

Nakagawa T, Sakamoto T, Hiraumi H, Kikkawa Y, Yamamoto N, Hamaguchi K, Ono K, Yamamoto M, Tabata Y, Teramukal S, Tanaka S, Tada H, Onodera R, Yonezawa A, K-I I, Ito J (2010) Topical insulin-like growth factor 1 treatment using gelatin hydrogels for glucocorticoid-resistant sudden sensorineural hearing loss: a prospective clinical trial. BMC Med 8:76

Noushi F, Richardson RT, Hardman J, Clark G, O’Leary S (2005) Delivery of neurotrophin- 3 to the cochlea using alginate beads. Otol Neurotol 26:528-533

O'Leary SJ, Richardson RR, McDermott HJ (2009) Principles of design and biological approaches for improving the selectivity of cochlear implant electrodes. J Neural Eng 6:055002

Pau HW, Just T, Bornitz M, Lasurashvilli N, Zahnert T (2007) Noise exposure of the inner ear during drilling: a cochleostomy for cochlear implantation. Laryngoscope 117:535-540

Pettingill LN, Minter RL, Shepherd RK (2008) Schwann cells genetically modified to express neurotrophins promote spiral ganglion neuron survival in vitro. Neuroscience 152:821-828

POLAK M (2004) Evaluation of hearing and auditory nerve function by combining ABR, DPOAE and eABR tests into a single recording session. J Neurosci Meth 134:141-149

Richardson RT, Wise A, O’Leary S, Hardman J, Casley D, Clark G (2004) Tracing neurotrophin-3 diffusion and uptake in the guinea pig cochlea. Hear Res 198:25-35

Richardson RT, Wise AK, Thompson BC, Flynn BO, Atkinson PJ, Fretwell NJ, Fallon JB, Wallace GG, Shepherd RK, Clark GM, 
O'Leary SJ (2009) Polypyrrole-coated electrodes for the delivery of charge and neurotrophins to cochlear neurons. Biomaterials 30:2614-2624

Rose CR, Blum R, Kafitz KW, Kovalchuk Y, Konnerth A (2004) From modulator to mediator: rapid effects of BDNF on ion channels. Bioessays 26:1185-1194

SaIjo S, Kimura RS (1984) Distribution of HRP in the inner ear after injection into the middle ear cavity. Acta Otolaryngol 97:593-610

ShePHeRd RK, Clark GM (1985) Progressive ototoxicity of neomycin monitored using derived brainstem response audiometry. Hear Res 18:105-110

Shepherd RK, Coco A, Epp SB, Crook JM (2005) Chronic depolarization enhances the trophic effects of brain-derived neurotrophic factor in rescuing auditory neurons following a sensorineural hearing loss. J Comp Neurol 486:145-158

SHEPHERd RK, Coco A, EpP SB (2008) Neurotrophins and electrical stimulation for protection and repair of spiral ganglion neurons following sensorineural hearing loss. Hear Res 242:100-109

Shinohara T, Bredberg G, Ulfendahl M, Рүyкko I, Olivius NP, Kaksonen R, Lindstrom B, Altschuler R, Miller JM (2002) Neurotrophic factor intervention restores auditory function in deafened animals. Proc Natl Acad Sci U S A 99:1657-1660

Shojt F, Miller AL, Mitchell A, Yamasoba T, Altschuler RA, Miller JM (2000) Differential protective effects of neurotrophins in the attenuation of noise-induced hair cell loss. Hear Res 146:134-142

Sly DJ, HefFer LF, White MW, ShePherd RK, Birch MG, Minter RL, Nelson NE, Wise AK, O'Leary SJ (2007) Deafness alters auditory nerve fibre responses to cochlear implant stimulation. Eur J Neurosci 26:510-522

Song BN, Li YX, HAN DM (2009) Delayed electrical stimulation and BDNF application following induced deafness in rats. Acta Otolaryngol 129:142-154

Staecker H, Kopke R, Malgrange B, Lefebvre P, Water TR (1996) NT-3 and/or BDNF therapy prevents loss of auditory neurons following loss of hair cells. Neuroreport 7:889-894

Staecker H, Gabaizadeh R, Federoff H, Van De Water TR (1998) Brain-derived neurotrophic factor gene therapy prevents spiral ganglion degeneration after hair cell loss. Otolaryngol Head Neck Surg 119:7-13

WANG Q GReEN SH (2011) Functional role of neurotrophin-3 in synapse regeneration by spiral ganglion neurons on inner hair cells after excitotoxic trauma in vitro. J Neurosci 31:7938-7949

Wise AK, Richardson R, Hardman J, Clark G, O’Leary S (2005) Resprouting and survival of guinea pig cochlear neurons in response to the administration of the neurotrophins brain-derived neurotrophic factor and neurotrophin-3. J Comp Neurol 487:147-165

Wise AK, Hume CR, Flynn BO, Jeelall YS, Suhr CL, Sgro BE, O'Leary SJ, SHEPHERd RK, Richardson RT (2010) Effects of localized neurotrophin gene expression on spiral ganglion neuron resprouting in the deafened cochlea. Mol Ther 18:1111-1122

Yamagata T, Miller JM, Ulfendahl M, Olivius NP, Altschuler RA, Pyкḱo I, Bredberg G (2004) Delayed neurotrophic treatment preserves nerve survival and electrophysiological responsiveness in neomycin-deafened guinea pigs. J Neurosci Res 78:75-86 